\title{
Atypical Celiac Disease: From Recognizing to Managing
}

\author{
B. Admou, ${ }^{1,2}$ L. Essaadouni, ${ }^{3}$ K. Krati, ${ }^{4}$ K. Zaher, ${ }^{2}$ M. Sbihi, ${ }^{5}$ L. Chabaa, ${ }^{6}$ \\ B. Belaabidia, ${ }^{7}$ and A. Alaoui-Yazidi ${ }^{2}$ \\ ${ }^{1}$ Laboratory of Immunology, Faculty of Medicine and University Hospital Center, BP 7010, Sidi Abbad, Marrakech, Morocco \\ ${ }^{2}$ Laboratory of Research "PCIM", Faculty of Medicine, University Cadi Ayyad, Marrakech, Morocco \\ ${ }^{3}$ Service of Internal Medicine, University Hospital Center, Marrakech, Morocco \\ ${ }^{4}$ Service of Gastroenterology, University Hospital Center, Marrakech, Morocco \\ ${ }^{5}$ Service of Peadiatrics, University Hospital Center, Marrakech, Morocco \\ ${ }^{6}$ Laboratory of Biochemistry, University Hospital Center, Marrakech, Morocco \\ ${ }^{7}$ Laboratory of Histopathology, University Hospital Center, Marrakech, Morocco
}

Correspondence should be addressed to B. Admou, admou.fmpm@gmail.com

Received 8 January 2012; Accepted 8 May 2012

Academic Editor: Govind K. Makharia

Copyright ( $\odot 2012$ B. Admou et al. This is an open access article distributed under the Creative Commons Attribution License, which permits unrestricted use, distribution, and reproduction in any medium, provided the original work is properly cited.

The nonclassic clinical presentation of celiac disease (CD) becomes increasingly common in physician's daily practice, which requires an awareness of its many clinical faces with atypical, silent, and latent forms. Besides the common genetic background (HLA DQ2/DQ8) of the disease, other non-HLA genes are now notably reported with a probable association to atypical forms. The availability of high-sensitive and specific serologic tests such as antitissue transglutuminase, antiendomysium, and more recent antideamidated, gliadin peptide antibodies permits to efficiently uncover a large portion of the submerged CD iceberg, including individuals having conditions associated with a high risk of developing CD (type 1 diabetes, autoimmune diseases, Down syndrome, family history of CD, etc.), biologic abnormalities (iron deficiency anemia, abnormal transaminase levels, etc.), and extraintestinal symptoms (short stature, neuropsychiatric disorders, alopecia, dental enamel hypoplasia, recurrent aphtous stomatitis, etc.). Despite the therapeutic alternatives currently in developing, the strict adherence to a GFD remains the only effective and safe therapy for CD.

\section{Introduction}

Celiac disease $(\mathrm{CD})$ is an intestinal chronic inflammatory and autoimmune disease that develops as a result of interplay between genetic, immunologic, and environmental factors [1]. Until recently, CD was considered to be a rare condition, with the highest incidence (1\% to $0.3 \%)$ in European countries $[2,3]$. The true incidence evaluated by a North American study is about $0.5 \%$ to $1 \%$, but many, if not most, of studied patients were asymptomatic members of high-risk groups [3, 4]. Recent epidemiological studies performed in North Africa and Asian areas also showed a high rate of CD: $0.53 \%$ in Egypt [5], $0.79 \%$ in Libya [6], $0.6 \%$ in Tunisia [7], $0.88 \%$ in Iran [8], $0.6 \%$ in Turkey [9], and $0.7 \%$ in India [10]. The classic form of CD typically presents in infancy and manifests as failure to thrive, diarrhea, abdominal distention, developmental delay, and, occasionally, severe malnutrition
$[11,12]$, which can lead to a true medical emergency [11]. Furthermore, serologic studies demonstrate that most celiac patients present with oligosymptomatic, latent, potential, and extraintestinal forms. These nonclassic clinical presentations become increasingly common and might reach about $50 \%$ of all diagnosed patients. The undiagnosed CD cases remain untreated, leaving individuals exposed to the risk of long-term complications, such as infertility, osteoporosis, or cancer [13-16].

Our aim is to emphasize the atypical clinical expression of celiac disease and suggest a diagnosis and managing approach.

\section{Genetic Background}

As demonstrated by several investigators, $\mathrm{CD}$ is one of the most common genetically based diseases; the part of genetic 


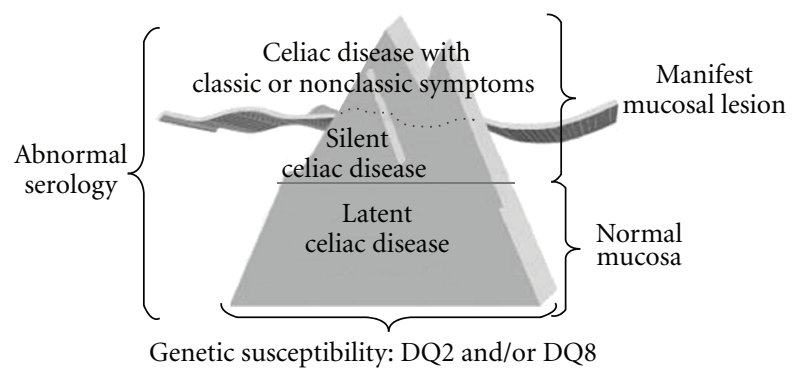

Figure 1: The celiac iceberg model [14].

background is fundamental in its pathogenesis, with possible influence of genetic factors on clinical and immunologic features [17-19]. Approximately $97 \%$ of individuals with CD have genetic markers on chromosome $6 \mathrm{p} 21$, called class II human leukocyte antigen (HLA). HLA DQ2 predominates, occurring in $90-95 \%$ of patients, and HLADQ8 occurs in the remainder $[11,18,20]$. Some studies also point to a correlation between DQ2 homozygousness and female sex, earlier age at diagnosis, shorter time span between onset of symptoms and diagnosis, and to a higher prevalence of classic clinical presentations among patients carrying double-dose $\mathrm{DQB1}{ }^{*} 02$ [21]. Other investigations suggest that MHC class I region plays a role in the development of diverse clinical forms of the disease [19, 22]. López-Vázquez et al. [22], thus showed that haplotype B8/DR3/DQ2 is notably overrepresented in atypical CD patients compared to typical ones $[19,22]$. In addition, similar studies displayed that MICA-A5.1 allele either is associated with atypical forms of CD in HLA-DQ2-negative patients or confers an additive effect to the DR3/DQ2 haplotype that may modulate the development of the disease [19, 23]. Also, linkage research pointed to chromosomal regions other than the HLA region, predisposing to CD with modest effects; the CTLA4 (cytotoxic T-lymphocyte associated), a closely located gene on chromosome $2 \mathrm{q} 33$, is one of these genes $[1,24]$. Alongside the HLA, recent genetic studies concerning potential CD patients identified a robust association on chromosome 4q27, involving IL-2, IL-21, and KIAA1109 gene cluster $[25,26]$, and also c-REL gene [26]. These facts might allow more understanding in CD pathogenesis.

\section{Clinical Faces of Celiac Disease}

Gee described the classical features of celiac disease in 1887 as diarrhea, lassitude, and failure to thrive [27], but the improvement of knowledge has subsequently disclosed several patterns of the disease [28]. A number of investigators believe that clinically apparent gluten-sensitive enteropathy represents the "tip of the iceberg" of the overall disease burden (Figure 1).

This concept demonstrates the clinical variability of $\mathrm{CD}$ and enlightens why the disease remains unidentified in a great proportion of individuals. In fact, the estimated ratio of diagnosed to undiagnosed individuals varies between $1: 5$ to $1: 8$ (the submerged part of the iceberg), usually because of atypical, minimal, or even absent complaints $[13,14]$.
Many authors defined atypical CD as follows:

(i) Atypical form. Absence or few gastrointestinal symptoms, presence of atypical symptoms, such as anemia due to iron deficiency, osteoporosis or osteopenia, infertility, low stature;

(ii) Silent form. Occasional diagnosis, histological or serological, in asymptomatic individuals;

(iii) Latent form, with 2 categories

(a) patients with previous $\mathrm{CD}$ diagnosis who responded to gluten-free diet (GFD) and presented a normal histology or only intraepithelial lymphocytes increase,

(b) individuals with normal intestinal mucosa, under diet including gluten, who will subsequently develop CD;

(iv) Refractory form. Patients with $\mathrm{CD}$ who do not respond to GFD $[12,14,29]$.

Patients with CD are diagnosed at any age and can exhibit a wide range of clinical manifestations (Table 1). In fact, beyond infancy, the symptoms of $\mathrm{CD}$ tend to be less dramatic $[30,31]$. Older children may present with constitutional short stature or dental enamel defects, and women comprise approximately $75 \%$ of newly diagnosed adult CD cases, with more clinically conspicuous disease [11,31].

Evidence suggests that the incidence of $\mathrm{CD}$ increases with age even in older patients [33]. Indeed, the majority of the elder cases remains undetected, often due to the absence of symptoms or because of atypical clinical presentations $[34,35]$. Osteoporosis represents one of the most frequent revealing circumstances of the disease in the elderly, and the rate of bone loss is accelerated in women after the menopause, likewise in men at the same age $[33,36]$. Anyway, physicians' lack of alertness in the older people may result in a significant delay in diagnosis, as CD is widely deemed to be a condition affecting younger subjects [33].

Moreover, a wider spectrum of neurologic syndromes may be the presenting extraintestinal manifestation of gluten sensitivity with or without intestinal pathology. These include headache, ataxia and psychiatric disorders [29], migraine, encephalopathy, chorea, brain stem dysfunction, myelopathy, mononeuritis multiplex, Guillain-Barrélike syndrome, and neuropathy with positive antiganglioside antibodies [37]. Additional studies showed high prevalence of gluten sensitivity in genetic neurodegenerative disorders such as hereditary spinocerebellar ataxia and Huntington's disease [37]. As well, oral manifestations, mostly recurrent apthous ulcers or stomatitis and dental enamel hypoplasia or defects, are atypical signs of $\mathrm{CD}$, and should be considered, even in the absence of any gastrointestinal symptom, at-risk subjects, and should therefore undergo diagnostic procedure for $\mathrm{CD}[28,38]$. Also, recurrent febrile infections associated to moderate neutropenia must be included in the diagnostic workup for atypical/silent $\mathrm{CD}$ in the general population [39]. Furthermore, many of biologic abnormalities either concur with $\mathrm{CD}$ or at times may reveal the disease such as 
TABLE 1: Clinical and biological revealing circumstances of atypical CD.

\begin{tabular}{l}
\hline Atypical clinical symptoms \\
\hline Anemia \\
Unclear vomiting \\
Constipation \\
Recurrent abdominal pain \\
Short stature \\
Irritability and impaired school performance \\
Impaired physical fitness and chronic fatigue \\
Osteopenia/osteoporosis/arthtritis \\
Dermatitis herpetiformis \\
Dental enamel hypoplasia \\
Recurrent aphtous stomatitis \\
Headache \\
Peripheral neuropathy \\
White matter lesions \\
Cerebellar ataxia \\
Epilepsy \\
Intracranial calcifications \\
Autism \\
Psychiatric disorders \\
Depression \\
Pubertal delay \\
Recurrent abortions \\
Infertility \\
\hline Biologic abnormalities \\
Anemia, iron deficiency; vitamin B12 and/or folate deficiency \\
Hypertransaminasemia \\
Hyperalkaline phosphatase level \\
Hyperalbuminemia \\
Hypercalcaemia, hypophosphatemia \\
Thrombocytosis, leukocytosis \\
\hline
\end{tabular}

anemia with iron, vitamin B12 and/or folate deficiencies, hypertransaminasemia (Table 1).

The prevalence of CD has increased sharply in recent years because of better recognition of the disease and its associated disorders (Table 2) [18, 36, 40]. A number of diseases seem to occur more commonly in CD. Many studies showed that patients with type 1 diabetes mellitus (T1DM), autoimmune thyroid disease, Sjögren's syndrome, primary biliary cirrhosis, Addison's disease, systemic lupus erythematosus, and alopecia areata may also exhibit similar genotypes of the celiac disease (HLA-DQ2 [DQA1*0501 and $\left.\left.\mathrm{DQB1}^{*} 0201\right]\right)$ and are at risk for gluten-sensitive enteropathy [11]. Autoimmune disorders occur 3 to 10 times more frequently in those with celiac disease than in the general population. Evidence exists that the risk of developing other autoimmune conditions increases with length of exposure to gluten [11, 18, 41]. Among associated CD conditions, T1DM is probably the most important; occurring in about
TABLE 2: List of possible celiac-disease-linked pathologies.

\begin{tabular}{l}
\hline Associated autoimmune diseases or other conditions \\
\hline Type 1 diabetes \\
Thyroid disorders (autoimmune or graves) \\
Liver disease (autoimmune hepatitis, primary biliary cirrhosis) \\
Myasthenia gravis \\
Primary biliary cirrhosis \\
Primary sclerosing cholangitis \\
Psoriasis \\
Sjögren disease \\
Systemic lupus erythematosus \\
Idiopathic dilated cardiomyopathy \\
Immunoglobulin A nephropathy \\
Lymphocytic or microscopic colitis \\
Autoimmune Addison's disease \\
Rheumatoid arthritis \\
Vitiligo or alopecia areata \\
\hline Associated genetic diseases \\
\hline Down syndrome \\
Turner syndrome \\
Williams syndrome \\
IgA deficiency \\
Commun variable immunodeficiency
\end{tabular}

$5 \%$ of CD patients $[40,42]$, with a large variance between ethnic populations (range: 0.97-16.4\%) [43]. In addition, unexplained and recurrent hypoglycemia in well managed diabetic individuals should alert the physician for $\mathrm{CD}$ screening [44]. Approximately 5\% of the patients with CD have thyroid disorders (either autoimmune (Hashimoto's) or Graves's disease) [42], and the ISPAD (International Society for Pediatric and Adolescent Diabetes) clinical practice consensus guidelines 2006-2007 recommend an assessment of the thyroid function at the diagnosis of $\mathrm{CD}$ and thereafter every second year in asymptomatic individuals and also a screening for $\mathrm{CD}$ at time of the diagnosis of these thyroid disorders and every second year thereafter $[45,46]$. Down or Turner syndromes also represent frequent linked conditions in which $\mathrm{CD}$ is often asymptomatic and then require systematic screening for $\mathrm{CD}[47,48]$. Furthermore, the association of some primary immunodeficiencies entities with CD has been described such as IgA deficiency [49] and common variable immunodeficiency [50].

\section{Serologic Testing: Performances and Limits}

Since the introduction of serological tests, and because of occasional screening, silent CD forms have been increasingly recognized. This is frequently the case of family predisposed individuals, and patients with associated autoimmune or genetic disorders. In $\mathrm{CD}$, highly sensitive and specific methods are nowadays widely used in laboratory testing such as antiendomysial (EMA) and antitissue transglutaminase (tTG) antibodies tests $[18,51]$. But, although these tests exhibit very high sensitivity and specificity $[11,13,32]$, 
TABLE 3: Characteristics of exclusive or combined serological tests used to detect CD $[11,13,18,32]$.

\begin{tabular}{lcccc}
\hline Serological tests & Sensitivity $(\%)$ & Specificity $(\%)$ & PPV $(\%)$ & NPV $(\%)$ \\
\hline IgG AGA & $57-78$ & $71-87$ & $20-90$ & $30-100$ \\
IgA AGA & $55-100$ & $65-100$ & $98-100$ & $70-100$ \\
IgA EMA & $86-100$ & $98-100$ & $>90$ & $80-95$ \\
IgA tTG & $90-96$ & $91-97$ & $>90$ & $>95$ \\
IgA tTG and EMA & $98-100$ & $98-100$ & 100 & 98 \\
IgA DGP & 98 & 100 & 94 & 97 \\
IgG DGP & 97 & 93 & 97 & 100 \\
IgA DGP + IgA tTG & 100 & 97 & 97 \\
IgG DGP + IgA tTG & 100 & 900 & 100 \\
\hline
\end{tabular}

IgG: immunoglobulin G; IgA: immunoglobulin A; AGA: antigliadin antibodies; EMA: endomysial antibodies; tTG: tissue transglutaminase; DGP: deamidated gliadin peptide; PPV: positive predictive value; NPV: negative predictive value.

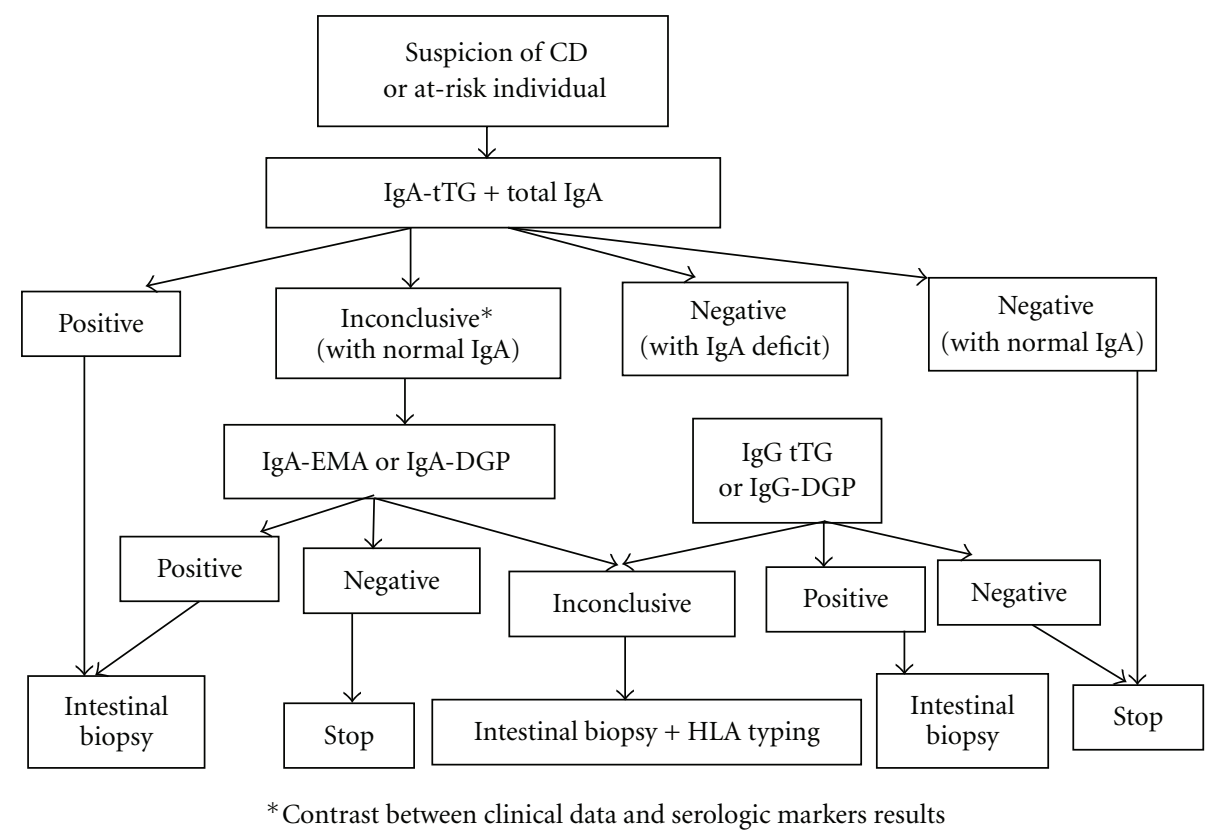

FIGURE 2: Algorithm proposal for biologic diagnosis of celiac disease.

recent investigations showed that their accuracy remains controversial in some conditions; sensitivity is considered unacceptable both in patients with minor degrees of mucosal damage and in cases with silent or oligosymptomatic forms [32]. Moreover, EMA and tTG have been found to be superior to AGA (anti-gliadin antibodies) tests $[11,13$, 18] and when used in combination have sensitivity and specificity greater than $95 \%[11,13]$. In addition, the recently developed deamidated gliadin peptide (DGP) antibody test shows promise in $\mathrm{CD}$ diagnostic $[32,51]$, and its performances are comparable to those of IgA-anti-tTG $[52,53]$. Moreover, IgG anti-DPG test has high diagnostic sensitivity not only in IgA-competent but also in IgA deficient CD patients [52]. Therefore, a combined evaluation of IgA-antitTG, and IgG anti-DPG seems to be adequate for serodiagnosis of $\mathrm{CD}$ irrespective of IgA deficiency and without the need for estimating total IgA concentrations $[52,53]$. The detection characteristics for AGA, EMA, tTG, and DGP tests are shown in Table 3. In practice, according to new recommendations, the initial serology testing consists on IgA-tTG screening, combined to total serum IgA measurement in order to rule out individuals with potential IgA deficiency. The serology test should be performed before eliminating gluten from patient's diet [54]. Actually, the biologic diagnosis should be improved by combining two performant serologic markers, such as IgA-tTG and -EMA or IgA-tTG and IgG-DGP according to suggested algorithm in Figure 2. The patients who test positive with these assays are consequently candidates for diagnostic endoscopy and smallbowel biopsy [51]. However, besides the atypical clinical expression of $\mathrm{CD}$, the diagnosis may be more difficult for many reasons: negative serology, irregular histological behavior, or inadequate number or place of biopsies [55].

Despite the evolving performances of these serologic testing, there are still significant problems concerning the diagnosis approach in some atypical conditions; for example, 
it has been proposed that IgG-AGA testing might be the best marker for neurological manifestations of gluten sensitivity, mainly for patients with sporadic ataxia $[60,61]$. Thus, in a recent study on gluten ataxia patients, Hadjivassiliou et al. [62], noticed anti-EMA antibodies in only $22 \%$ of patients, and anti-TG2 IgA in up to $38 \%$ of cases, but often at lower titres than those seen in patients with gluten sensitivity enteropathy [62]. On the other hand, the serology is generally thought to be unreliable in children $<18$ months of age [63]. This is due to a number of factors including the high proportion of children on breast milk, lower IgA levels, and the under-developed immune system. Some authors have suggested that IgA-AGA may be useful in this situation. This view is supported by a recent study carried out in 208 children $<18$ months of age diagnosed with CD [64], showing a better sensitivity of IgA-AGA compared to both the IgA-tTG and IgA EMA [65, 66].

\section{Seronegative Celiac Disease}

Not all patients have positive CD serologic markers at presentation $[67,68]$. In fact, the presence of related CD antibodies correlates with the degree of villous atrophy and possibly the mode of presentation of the disease $[67,69]$. Patients with lesser degrees of villous atrophy are less likely to have positive celiac serology [18], and patients who present persistently positive serology and negative biopsy probably have latent CD [12]. Moreover, children younger than 2 years of age lack EMA and tTG antibodies; for this reason, serological testing in children younger than 5 years of age may be less reliable and requires additional investigation [18]. On the other hand, in individuals who are IgA-deficient, the measurement of IgG-EMA and anti-tTG offers reliable results with excellent sensitivity (close to 100\%) and specificity $[12,18]$. Anyway, if CD suspicion is high with persistently negative tests, individuals must perform typing for HLA and, if positive, they must perform duodenal biopsy or alternatively perform biopsy directly $[12,55]$.

\section{Histopathologic Findings}

The intestinal biopsy represents the gold standard diagnosis for CD [12, 55]. According to Marsh-Oberhuber's [56, 57] criteria (Table 4), the spectrum of alterations compatible with CD consists of intraepithelial lymphocytic (IEL) infiltration, pattern of crypts, and villous atrophy, and patient's symptoms frequently correlate with the degree of tissue injury [59]. However, IEL increase with normal mucosa architecture may be observed in autoimmune diseases, such as SLE, rheumatoid arthritis, and Hashimoto's thyroiditis, in patients using nonhormonal anti-inflammatory treatment, in CD's initial presentation, and latent $\mathrm{CD}[55,70]$. An increase in IEL may also reflect a state of $\mathrm{T}$ cells activation triggered by gluten, immune abnormalities, drugs, and infectious agents. Celiac patients, who present only IEL increase with no alterations in the architecture of the mucosa, may be symptomatic and be under increased complications risk [12]. Similarly, villous atrophy may be due to other causes such as Crohn's disease, collagenous sprue, and autoimmune
TABLE 4: Histopathologic classification of CD based on MarshOberhuber [56, 57], and Corazza and Villanacci [58] new grading system $[12,57,59]$.

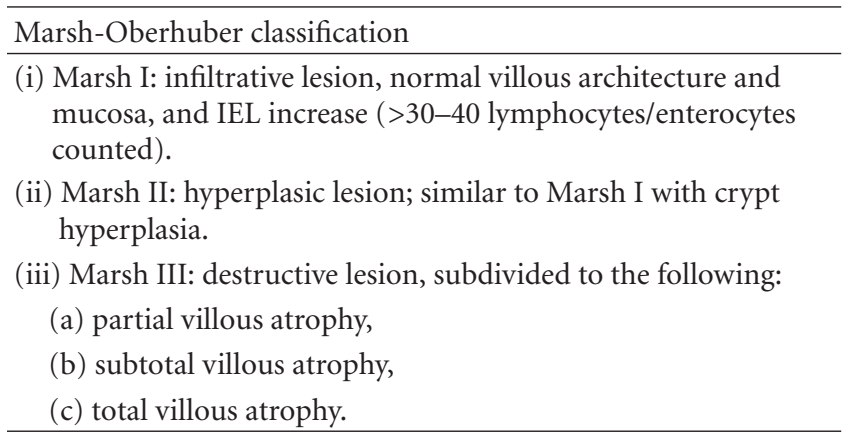

New grading system

(i) Grade A (nonatrophic): > 25 IELs/100 enterocytes.

(ii) Grade B (atrophic): villous-crypt ratio <3: 1 .

(iii) Grade B2 (atrophic): no detectable villi.

enteropathy [71]. Moreover, a recent prospective evaluation led by different expert pathologists highlighted that a recently proposed three-grade classification system [58] gives better interobserver agreement as compared with the established six-grade Marsh-Oberhuber classification (Table 4) [72].

Similarly to wide variation in clinical manifestations, GSE has a wide spectrum of histological abnormalities, which makes interpretation of small-intestinal biopsy specimens problematic for the pathologist [73]. Therefore, it is not advised to affirm a diagnosis based only on the histological findings, because the disease does not compromise uniformly intestine, and alterations are not observed exclusively in $\mathrm{CD}[12,55]$. Actually, many differential diagnoses (Table 5) may give rise to $\mathrm{CD}$, making the diagnosis more difficult.

\section{HLA Typing}

All CD patients carry HLA-DQ2 or HLA-DQ8 [20]. However, up to $40 \%$ of the general population also carries these HLA haplotypes. Their presence is necessary for the development of celiac disease, but the absence of these alleles virtually excludes the diagnosis [18] with a negative predictive value for CD close to $100 \%$ [20]. HLA typing represents the first step for investigating relatives of $\mathrm{CD}$ patients, specifically 1st-degree relatives and then permits to identify individuals for evaluation with biopsy [12]. In practice, if CD suspicion is high, with persistently negative tests, individuals must perform typing for HLA and, if positive, they must perform duodenal biopsy or alternatively perform biopsy directly. Likewise, HLA typing is indicated in individuals who refuse to undergo biopsy [12].

\section{Gluten-Free Diet: Indications and Managing}

An increased incidence of small-bowel malignancies, adenocarcinoma, and enteropathy-associated T-cell lymphoma has been reported in untreated CD $[18,74]$. 
TABle 5: Celiac disease differential diagnosis [12].

Anorexia nervosa
Autoimmune enteropathy
Bacterial overgrowth
Collagenous sprue
Crohn's disease
Giardiasis
HIV enteropathy
Hipogammaglobulinemia
Gastroenterite infecciosa
Intestinal lymphoma
Radiation enteritis
Ischemic enteritis
Lactose intolerance
Common variable immunodeficiency
Soy protein intolerance
Tropical sprue
Tuberculosis
Whipple's disease
Zolliger-Ellison syndrome
Eosinophilic gastroenteritis

TABLE 6: Indications of GFD in CD of children and adolescents [75].

\begin{tabular}{ll}
\hline CD clinical form & Indications of GFD \\
\hline Symptomatic & Therapeutic \\
Silent & Preventive: may be discussed \\
Latent & Surveillance \\
\hline
\end{tabular}

A strict and lifelong gluten-free diet (GFD) has been demonstrated to be effective and safe, preventing most potential complications of the disease, including autoimmune disease, osteoporosis, infertility, prematurity, and malignancy [76, 77]. However, there is still no evidence that patients who have symptom-free celiac disease are at increased risk of small-intestinal lymphoma or other complications [71]. On the other hand, diet trials in patients with gluten sensitivity and neurologic syndromes have shown variable results and have been inconclusive in some neurologic diseases such as autism and schizophrenia [37]. Furthermore, in asymptomatic patients, a second follow-up biopsy under a GFD is advised to demonstrate the histological recovery of the mucosa, which usually does not develop before six months [73].

In general, the guidance of GFD may be envisaged according to three modalities (Table 6).

(i) Typical or symptomatic CD; GFD is a formal therapeutic indication.

(ii) Silent CD; GFD is discussed under two circumstances.

(iii) Silent CD discovered on the occasion of a serological screening in the family of a celiac or in a patient at risk (diabetes mellitus, dermatitis herpetiforme); in this case, the lesser clinical or nutritional sign would treat the subject as symptomatic and plead in favor of GFD.

(iv) CD becoming silent in the second childhood after that the active disease in the first childhood was treated several years by a well monitoring GFD.

In these two situations and in individual really clinically and biologically asymptomatic, the decision to introduce or to resume the GFD is then rather preventive.

(v) Latent CD (subjects genetically predisposed with normal intestinal mucosa); a simple clinical and biological surveillance is advocated by recent studies [75].

Beside the GFD, the management of many of CD-linked features may require additional supplementation particularly in nutritional problems, such as lower $\mathrm{Hb}$ and low $\mathrm{Fe}$, low albumin or $\mathrm{Ca}$, cholesterol and folates disorders [32, 78-80]. Likewise, in CD patients with low bone mineral density, apart from a GFD, a rational managing should follow conventional lines, including increasing exercise, stopping smoking, and avoiding alcohol excess and ensuring an adequate $\mathrm{Ca}$ intake using supplements if necessary [36]. In addition, newly therapeutic alternatives are currently interested in the pathogenesis of the disease, focusing on engineering gluten-free grains, degradation of immunodominant gliadin peptides that resist intestinal proteases by exogenous endopeptidases, decrease in intestinal permeability by blockage of the epithelial zonuline receptor, inhibition of intestinal tTG2 activity by transglutaminase inhibitors, inhibition of gluten peptide presentation by HLA-DQ2 antagonists, modulation or inhibition of proinflammatory cytokines, and induction of oral tolerance to gluten $[14,81,82]$. But, at this time, strict adherence to a GFD remains the only effective and safe therapy for CD [14].

\section{Refractory Celiac Disease}

A small proportion of CD patients fails to improve after a GFD and may be considered as atypical regarding their outcome $[14,83]$. Refractory celiac disease (RCD) was recently defined as persisting or recurring villous atrophy with crypt hyperplasia and increased intraepithelial lymphocytes (IELs) in spite of a strict GFD for more than 12 months [71, 84]. It can be either primary, as lack of initial response to diet, or secondary, as unresponsiveness to diet in the form of a relapse [73]. Two categories of RCD are recently being recognized: type I without aberrant T cells and type II with aberrant $\mathrm{T}$ cells [85]. The presence of an aberrant clonal intraepithelial T-cell population and/or loss of antigen on IELs seem to characterize population on high risk for development of overt lymphoma and differentiates RCD II from RCD I, which shows low or almost absent aberrant T cells [84].

To manage RCD, Krauss and Schuppan [71] recommend firstly to reassess the diagnosis of CD in order to exclude other diseases, such as giardiasis, tropical sprue, postinfectious diarrhea, collagenous sprue, protein intolerance or protein-losing enteropathy, tuberculosis (including atypical), AIDS, common variable immunodeficiency syndrome, 
Whipple's disease, ulcerative jejunitis, lymphocytic colitis, radiation enteritis, immunoproliferative small-intestinal disease, Crohn's disease, eosinophilic gastroenteritis, and autoimmune enteropathy [71, 84], and then to check for errors in diet or compliance [71]. The treatment of RCD I consists of a first-line immunosuppressive therapy based on azathioprine after induction of clinical remission with corticosteroids [86]. A second-line therapy (Cyclosporine A, infliximab, tacrolimus) is suggested in case of clinical deterioration despite corticosteroid therapy or intolerance to azathioprine [87] RCD II is usually resistant to medical therapies, and facing persistent clinical symptoms and/or a high percentage of aberrant $\mathrm{T}$ cells in intestinal biopsies in spite of a corticosteroid treatment, more aggressive therapeutic schemes should be considered [84].

\section{Conclusion}

Celiac disease represents a prototype of disease from which science and medicine take advantage, offering more and uninterrupted understandings both in genetic, clinic, diagnosis, and management aspects. Against its potential complications, the real challenge is to recognize asymptomatic or oligosymptomatic CD cases. The diagnosis should also be improved by a process of case finding focused on at-risk groups.

\section{References}

[1] L. M. Sollid, S. N. McAdam, O. Molberg et al., "Genes and environment in celiac disease," Acta Odontologica Scandinavica, vol. 59, no. 3, pp. 183-186, 2001.

[2] M. Mäki, K. Mustalahti, J. Kokkonen et al., "Prevalence of celiac disease among children in Finland," New England Journal of Medicine, vol. 348, no. 25, pp. 2517-2524, 2003.

[3] G. Telega, T. R. Bennet, and S. Werlin, "Emerging new clinical patterns in the presentation of celiac disease," Archives of Pediatrics and Adolescent Medicine, vol. 162, no. 2, pp. 164-168, 2008.

[4] E. J. Hoffenberg, T. MacKenzie, K. J. Barriga et al., "A prospective study of the incidence of childhood celiac disease," Journal of Pediatrics, vol. 143, no. 3, pp. 308-314, 2003.

[5] M. Abu-Zekry, D. Kryszak, M. Diab, C. Catassi, and A. Fasano, "Prevalence of celiac disease in egyptian children disputes the east west agriculture-dependent spread of the disease," Journal of Pediatric Gastroenterology and Nutrition, vol. 47, no. 2, pp. 136-140, 2008.

[6] K. Alarida, J. Harown, A. Ahmaida et al., "Coeliac disease in Libyan children: a screening study based on the rapid determination of anti-transglutaminase antibodies," Digestive and Liver Disease, vol. 43, no. 9, pp. 688-691, 2011.

[7] M. B. Hariz, M. Kallel-Sellami, L. Kallel et al., "Prevalence of celiac disease in Tunisia: mass-screening study in school children," European Journal of Gastroenterology and Hepatology, vol. 19, no. 8, pp. 687-694, 2007.

[8] F. Imanzadeh, A. A. Sayyari, M. Yaghoobi, M. R. Akbari, H. Shafagh, and A. R. Farsar, "Celiac disease in children with diarrhea is more frequent than previously suspected," Journal of Pediatric Gastroenterology and Nutrition, vol. 40, no. 3, pp. 309-311, 2005.
[9] V. Ertekin, M. A. Selimoglu, F. Kardas, and E. Aktas, "Prevalence of CD in Turkish children," Journal of Clinical Gastroenterology, vol. 39, pp. 689-691, 2005.

[10] A. Sood, V. Midha, N. Sood, G. Avasthi, and A. Sehgal, "Prevalence of celiac disease among school children in Punjab, North India," Journal of Gastroenterology and Hepatology, vol. 21, no. 10, pp. 1622-1625, 2006.

[11] D. A. Nelsen, "Gluten-sensitive enteropathy (celiac disease): more common than you think," American Family Physician, vol. 66, no. 12, pp. 2259-2266, 2002.

[12] T. S. G. Silva and T. W. Furlanetto, "Diagnosis of celiac disease in adults," Revista da Associacao Medica Brasileira, vol. 56, no. 1, pp. 122-126, 2010.

[13] J. S. Leeds, A. D. Hopper, and D. S. Sanders, "Coeliac disease," British Medical Bulletin, vol. 88, no. 1, pp. 157-170, 2008.

[14] E. Lionetti and C. Catassi, "New clues in celiac disease epidemiology, pathogenesis, clinical manifestations, and treatment," International Reviews of Immunology, vol. 30, no. 4, pp. 219-231, 2011.

[15] A. Ferguson and K. Kingstone, "Coeliac disease and malignancies," Acta Paediatrica, International Journal of Paediatrics, Supplement, vol. 85, no. 412, pp. 78-81, 1996.

[16] J. Cosnes, C. Cosnes, A. Cosnes et al., "Undiagnosed celiac disease in childhood," Gastroenterologie Clinique et Biologique, vol. 26, no. 6-7, pp. 616-623, 2002.

[17] P. H. R. Green and B. Jabri, "Coeliac disease," The Lancet, vol. 362, no. 9381, pp. 383-391, 2003.

[18] M. M. Niewinski, "Advances in Celiac disease and gluten-free diet," Journal of the American Dietetic Association, vol. 108, no. 4, pp. 661-672, 2008.

[19] A. Lopez-Vazquez, L. Rodrigo, D. Fuentes et al., "MHC class I chain related gene A (MICA) modulates the development of coeliac disease in patients with the high risk heterodimer DQA1*0501/DQB1*0201," Gut, vol. 50, no. 3, pp. 336-340, 2002.

[20] V. M. Wolters and C. Wijmenga, "Genetic background of celiac disease and its clinical implications," American Journal of Gastroenterology, vol. 103, no. 1, pp. 190-195, 2008.

[21] P. Zubillaga, M. C. Vidales, I. Zubillaga, V. Ormaechea, N. García-Urkía, and J. C. Vitoria, "HLA-DQA1 and HLA-DQB1 genetic markers and clinical presentation in celiac disease," Journal of Pediatric Gastroenterology and Nutrition, vol. 34, no. 5, pp. 548-554, 2002.

[22] A. López-Vázquez, D. Fuentes, L. Rodrigo et al., "MHC class I region plays a role in the development of diverse clinical forms of celiac disease in a Saharawi population," American Journal of Gastroenterology, vol. 99, no. 4, pp. 662-667, 2004.

[23] A. Lopez-Vazquez, L. Rodrigo, D. Fuentes et al., "MICA-A5.1 allele is associated with atypical forms of celiac disease in HLADQ2-negative patients," Immunogenetics, vol. 53, no. 10-11, pp. 989-991, 2002.

[24] A. T. Naluai, S. Nilsson, L. Samuelsson et al., "The CTLA4/ CD28 gene region on chromosome 2 q33 confers susceptibility to celiac disease in a way possibly distinct from that of type 1 diabetes and other chronic inflammatory disorders," Tissue Antigens, vol. 56, no. 4, pp. 350-355, 2000.

[25] D. A. Van Heel, L. Franke, K. A. Hunt et al., "A genome-wide association study for celiac disease identifies risk variants in the region harboring IL2 and IL21," Nature Genetics, vol. 39, no. 7, pp. 827-829, 2007.

[26] M. P. Sperandeo, A. Tosco, V. Izzo et al., "Potential celiac patients: a model of celiac disease pathogenesis," PLoS ONE, vol. 6, no. 7, Article ID e21281, 2011. 
[27] S. Gee, "On the celiac affection," Saint Bartholomew's Hospital Reports, vol. 24, pp. 17-20, 1988.

[28] L. Pastore, M. De Benedittis, M. Petruzzi et al., "Importance of oral signs in the diagnosis of atypical forms of celiac disease," Recenti Progressi in Medicina, vol. 95, no. 10, pp. 482-490, 2004.

[29] M. Spina, G. Incorpora, T. Trigilia, F. Branciforte, G. Franco, and F. Di Gregorio, "Headache as atypical presentation of celiac disease: report of a clinical case," Pediatria Medica $e$ Chirurgica, vol. 23, no. 2, pp. 133-135, 2001.

[30] C. Feighery, “Coeliac disease," British Medical Journal, vol. 319, no. 7204, pp. 236-239, 1999.

[31] C. Ciacci, M. Cirillo, R. Sollazzo, G. Savino, F. Sabbatini, and G. Mazzacca, "Gender and clinical presentation in adult celiac disease," Scandinavian Journal of Gastroenterology, vol. 30, no. 11, pp. 1077-1081, 1995.

[32] S. Niveloni, E. Sugai, A. Cabanne et al., "Antibodies against synthetic deamidated gliadin peptides as predictors of celiac disease: prospective assessment in an adult population with a high pretest probability of disease," Clinical Chemistry, vol. 53, no. 12, pp. 2186-2192, 2007.

[33] A. Vilppula, K. Kaukinen, L. Luostarinen et al., "Increasing prevalence and high incidence of celiac disease in elderly people: a population-based study," BMC Gastroenterology, vol. 9, article 49, 2009.

[34] G. L. Hankey and G. K. T. Holmes, "Coeliac disease in the elderly," Gut, vol. 35, no. 1, pp. 65-67, 1994.

[35] Y. Lurie, D. A. Landau, J. Pfeffer, and R. Oren, "Celiac disease diagnosed in the elderly," Journal of Clinical Gastroenterology, vol. 42, no. 1, pp. 59-61, 2008.

[36] A. Fasano, I. Berti, T. Gerarduzzi et al., "Prevalence of Celiac disease in at-risk and not-at-risk groups in the United States: a large multicenter study," Archives of Internal Medicine, vol. 163, no. 3, pp. 286-292, 2003.

[37] K. O. Bushara, "Neurologic presentation of celiac disease," Gastroenterology, vol. 128, no. 4, supplement 1, pp. S92-S97, 2005.

[38] J. Cheng, T. Malahias, P. Brar, M. T. Minaya, and P. H. R. Green, "The Association between celiac disease, dental enamel defects, and aphthous ulcers in a United States cohort," Journal of Clinical Gastroenterology, vol. 44, no. 3, pp. 191-194, 2010.

[39] S. Leonardi, G. Vitaliti, and M. La Rosa, "Recurrent febrile infections and neutropenia in a child with silent celiac disease," Clinical Pediatrics, vol. 49, no. 2, pp. 146-149, 2010.

[40] N. Chand and A. A. Mihas, "Celiac disease: current concepts in diagnosis and treatment," Journal of Clinical Gastroenterology, vol. 40, no. 1, pp. 3-14, 2006.

[41] M. Viljamaa, K. Kaukinen, H. Huhtala, S. Kyrönpalo, M. Rasmussen, and P. Collin, "Coeliac disease, autoimmune diseases and gluten exposure," Scandinavian Journal of Gastroenterology, vol. 40, no. 4, pp. 437-443, 2005.

[42] N. McGough and J. H. Cummings, "Coeliac disease: a diverse clinical syndrome caused by intolerance of wheat, barley and rye," Proceedings of the Nutrition Society, vol. 64, no. 4, pp. 434-450, 2005.

[43] G. K. T. Holmes, "Screening for coeliac disease in type 1 diabetes," Archives of Disease in Childhood, vol. 87, no. 6, pp. 495-499, 2002.

[44] D. Iafusco, F. Rea, F. Prisco, and J. F. Fitzgerald, "Hypoglycemia and reduction of the insulin requirement as a sign of celiac disease in children with IDDM," Diabetes Care, vol. 21, no. 8, pp. 1379-1381, 1998.

[45] O. Kordonouri, A. M. Maguire, M. Knip et al., "Other complications and associated conditions," Pediatric Diabetes, vol. 8, no. 3, pp. 171-176, 2007.
[46] E. E. Fröhlich-Reiterer, S. Hofer, S. Kaspers et al., "Screening frequency for celiac disease and autoimmune thyroiditis in children and adolescents with type 1 diabetes mellitus-data from a German/Austrian multicentre survey," Pediatric Diabetes, vol. 9, no. 6, pp. 546-553, 2008.

[47] M. Bonamico, A. M. Pasquino, P. Mariani et al., "Prevalence and clinical picture of celiac disease in Turner syndrome," Journal of Clinical Endocrinology and Metabolism, vol. 87, no. 12, pp. 5495-5498, 2002.

[48] R. M. Nisihara, L. M. S. Kotze, S. R. R. Utiyama, N. P. Oliveira, P. T. Fiedler, and I. T. Messias-Reason, "Celiac disease in children and adolescents with Down syndrome," Jornal de Pediatria, vol. 81, no. 5, pp. 373-376, 2005.

[49] F. Cataldo, V. Marino, A. Ventura, G. Bottaro, and G. R. Corazza, "Prevalence and clinical features of selective immunoglobulin A deficiency in coeliac disease: an Italian multicentre study," Gut, vol. 42, no. 3, pp. 362-365, 1998.

[50] D. Béchade, J. Desramé, G. De Fuentés, P. Camparo, J. J. Raynaud, and J. P. Algayres, "Common variable immunodeficiency and celiac disease," Gastroenterologie Clinique et Biologique, vol. 28, no. 10, pp. 909-912, 2004.

[51] K. Lindfors, O. Koskinen, and K. Kaukinen, "An update on the diagnostics of celiac disease," International Reviews of Immunology, vol. 30, no. 4, pp. 185-196, 2011.

[52] D. Villalta, E. Tonutti, C. Prause et al., "IgG antibodies against deamidated gliadin peptides for diagnosis of celiac disease in patients with IgA deficiency," Clinical Chemistry, vol. 56, no. 3 , pp. 464-468, 2010.

[53] C. Prause, T. Richter, S. Koletzko et al., "New developments in serodiagnosis of childhood celiac disease: assay of antibodies against deamidated gliadin," Annals of the New York Academy of Sciences, vol. 1173, pp. 28-35, 2009.

[54] D. P. Westerberg, J. M. Gill, B. Dave, M. J. DiPrinzio, A. Quisel, and A. Foy, "New strategies for diagnosis and management of celiac disease," Journal of the American Osteopathic Association, vol. 106, no. 3, pp. 145-151, 2006.

[55] M. F. Kagnoff, "AGA institute medical position statement on the diagnosis and management of celiac disease," Gastroenterology, vol. 131, no. 6, pp. 1977-1980, 2006.

[56] M. N. Marsh, "Gluten, major histocompatibility complex, and the small intestine: a molecular and immunobiologic approach to the spectrum of gluten sensitivity ('celiac sprue')," Gastroenterology, vol. 102, no. 1, pp. 330-354, 1992.

[57] G. Oberhuber, G. Granditsch, and H. Vogelsang, "The histopathology of coeliac disease: time for a standardized report scheme for pathologists," European Journal of Gastroenterology and Hepatology, vol. 11, no. 10, pp. 1185-1194, 1999.

[58] G. R. Corazza and V. Villanacci, "Coeliac disease," Journal of Clinical Pathology, vol. 58, no. 6, pp. 573-574, 2005.

[59] A. Rostom, J. A. Murray, and M. F. Kagnoff, "American gastroenterological association (AGA) institute technical review on the diagnosis and management of celiac disease," Gastroenterology, vol. 131, no. 6, pp. 1981-2002, 2006.

[60] M. Hadjivassiliou, R. Grünewald, B. Sharrack et al., "Gluten ataxia in perspective: epidemiology, genetic susceptibility and clinical characteristics," Brain, vol. 126, no. 3, pp. 685-691, 2003.

[61] M. T. Pellecchia, R. Scala, A. Filla, G. De Michelle, C. Ciacci, and P. Barone, "Idiopathic cerebellar ataxia associated with celiac disease: lack of distinctive neurological features," Journal of Neurology Neurosurgery and Psychiatry, vol. 66, no. 1, pp. 32-35, 1999. 
[62] M. Hadjivassiliou, D. S. Sanders, N. Woodroofe, C. Williamson, and R. A. Grünewald, "Gluten ataxia," Cerebellum, vol. 7, no. 3, pp. 494-498, 2008.

[63] R. Steele, "for the CRF* (Coeliac Research Fund Clinical Advisory Committee Members) Diagnosis and management of coeliac disease in children," Postgraduate Medical Journal, vol. 87, pp. 19-25, 2011.

[64] C. Lagerqvist, I. Dahlbom, T. Hansson et al., "Antigliadin immunoglobulin a best in finding celiac disease in children younger than 18 months of age," Journal of Pediatric Gastroenterology and Nutrition, vol. 47, no. 4, pp. 428-435, 2008.

[65] M. Stern, "Comparative evaluation of serologic tests for celiac disease: a European initiative toward standardization," Journal of Pediatric Gastroenterology and Nutrition, vol. 31, no. 5, pp. 513-519, 2000.

[66] R. Berger and G. Schmidt, "Evaluation of six anti-gliadin antibody assays," Journal of Immunological Methods, vol. 191, no. 1, pp. 77-86, 1996.

[67] J. A. Abrams, B. Diamond, H. Rotterdam, and P. H. R. Green, "Seronegative celiac disease: increased prevalence with lesser degrees of villous atrophy," Digestive Diseases and Sciences, vol. 49, no. 4, pp. 546-550, 2004.

[68] W. Dickey, S. A. McMillan, and D. F. Hughes, "Sensitivity of serum tissue transglutaminase antibodies for endomysial antibody positive and negative coeliac disease," Scandinavian Journal of Gastroenterology, vol. 36, no. 5, pp. 511-514, 2001.

[69] A. Tursi, G. Brandimarte, and G. M. Giorgetti, "Prevalence of antitissue transglutaminase antibodies in different degrees of intestinal damage in celiac disease," Journal of Clinical Gastroenterology, vol. 36, no. 3, pp. 219-221, 2003.

[70] S. Kakar, V. Nehra, J. A. Murray, G. A. Dayharsh, and L. J. Burgart, "Significance of intraepithelial lymphocytosis in small bowel biopsy samples with normal mucosal architecture," American Journal of Gastroenterology, vol. 98, no. 9, pp. 20272033, 2003.

[71] N. Krauss and D. Schuppan, "Monitoring nonresponsive patients who have celiac disease," Gastrointestinal Endoscopy Clinics of North America, vol. 16, no. 2, pp. 317-327, 2006.

[72] M. J. Armstrong, G. G. Robins, and P. D. Howdle, "Recent advances in coeliac disease," Current Opinion in Gastroenterology, vol. 25, no. 2, pp. 100-109, 2009.

[73] A. Ensari, "Gluten-sensitive enteropathy (celiac disease): controversies in diagnosis and classification," Archives of Pathology and Laboratory Medicine, vol. 134, no. 6, pp. 826-836, 2010.

[74] T. R. Card, J. West, and G. K. T. Holmes, "Risk of malignancy in diagnosed coeliac disease: a 24-year prospective, population-based, cohort study," Alimentary Pharmacology and Therapeutics, vol. 20, no. 7, pp. 769-775, 2004.

[75] J. Schmitz, "Le régime sans gluten chez l'enfant," Journal de Pédiatrie et de Puériculture, vol. 20, pp. 337-344, 2007.

[76] K. P. Zimmer, "Nutrition and celiac disease," Current Problems in Pediatric and Adolescent Health Care, vol. 41, no. 9, pp. 244247, 2011.

[77] D. Schuppan, M. D. Dennis, and C. P. Kelly, "Celiac disease: epidemiology, pathogenesis, diagnosis, and nutritional management," Nutrition in Clinical Care, vol. 8, no. 2, pp. 54-69, 2005.

[78] E. M. Scott, B. B. Scott, and I. Gaywood, "Guidelines for osteoporosis in coeliac disease and inflammatory bowel disease," Gut, vol. 46, no. 1, pp. i1-i8, 2000.

[79] A. K. Patwari, V. K. Anand, G. Kapur, and S. Narayan, "Clinical and nutritional profile of children with celiac disease," Indian Pediatrics, vol. 40, no. 4, pp. 337-342, 2003.
[80] U. Volta, L. De Franceschi, F. Lari, N. Molinaro, M. Zoli, and F. B. Bianchi, "Coeliac disease hidden by cryptogenic hypertransaminasaemia," The Lancet, vol. 352, no. 9121, pp. 26-29, 1998.

[81] D. Schuppan, Y. Junker, and D. Barisani, "Celiac disease: from pathogenesis to novel therapies," Gastroenterology, vol. 137, no. 6, pp. 1912-1933, 2009.

[82] A. Lerner, "New therapeutic strategies for celiac disease," Autoimmunity Reviews, vol. 9, no. 3, pp. 144-147, 2010.

[83] S. Daum, C. Cellier, and C. J. J. Mulder, "Refractory coeliac disease," Best Practice and Research, vol. 19, no. 3, pp. 413-424, 2005.

[84] A. Al-Toma, W. H. M. Verbeek, and C. J. J. Mulder, "The management of complicated celiac disease," Digestive Diseases, vol. 25, no. 3, pp. 230-236, 2007.

[85] C. Cellier, E. Delabesse, C. Helmer et al., "Refractory sprue, coeliac disease, and enteropathy-associated T-cell lymphoma," The Lancet, vol. 356, no. 9225, pp. 203-208, 2000.

[86] M. S. Goerres, J. W. R. Meijer, P. J. Wahab et al., "Azathioprine and prednisone combination therapy in refractory coeliac disease," Alimentary Pharmacology and Therapeutics, vol. 18, no. 5, pp. 487-494, 2003.

[87] P. J. Wahab, J. B. A. Crusius, J. W. R. Meijer, J. J. Uil, and C. J. J. Mulder, "Cyclosporin in the treatment of adults with refractory coeliac disease - an open pilot study," Alimentary Pharmacology and Therapeutics, vol. 14, no. 6, pp. 767-774, 2000. 


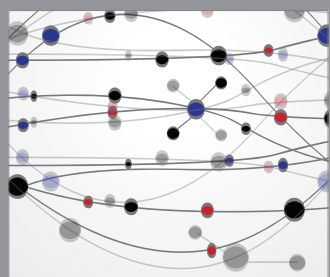

The Scientific World Journal
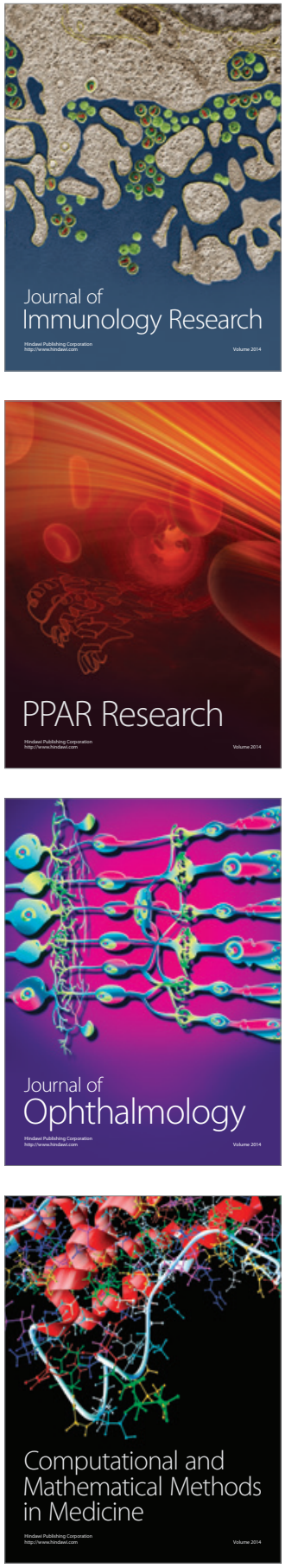

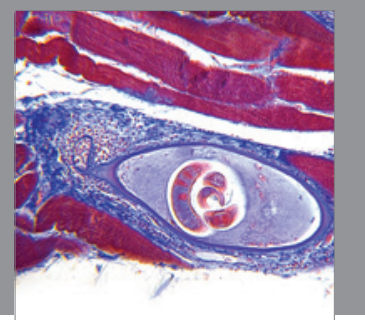

Gastroenterology

Research and Practice
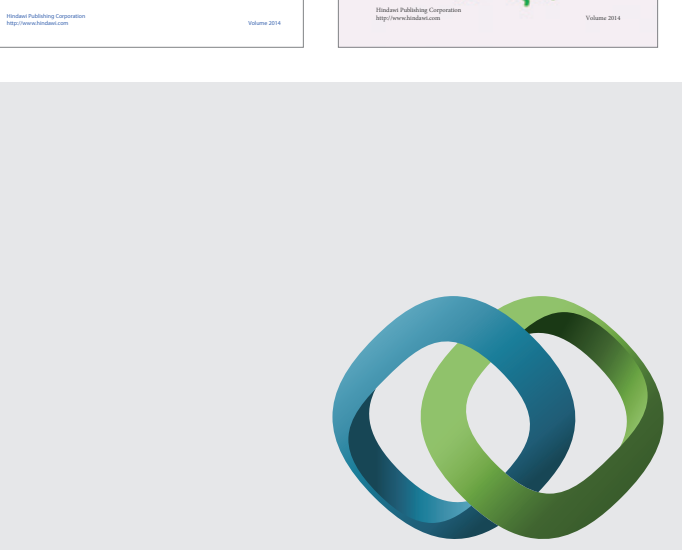

\section{Hindawi}

Submit your manuscripts at

http://www.hindawi.com
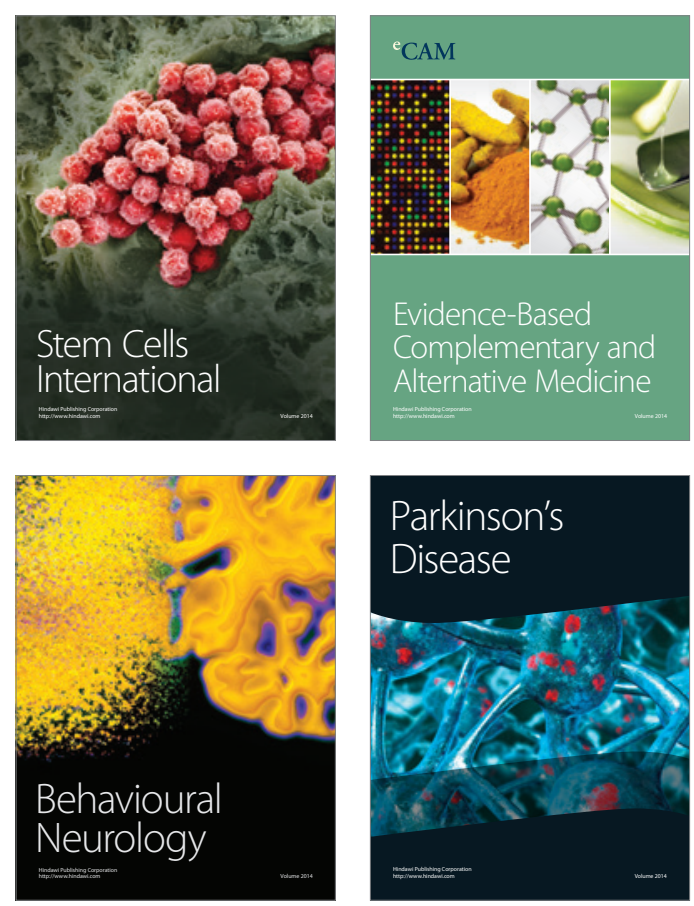

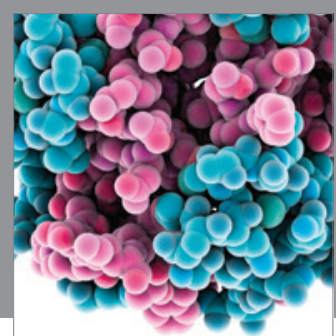

Journal of
Diabetes Research

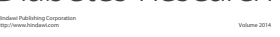

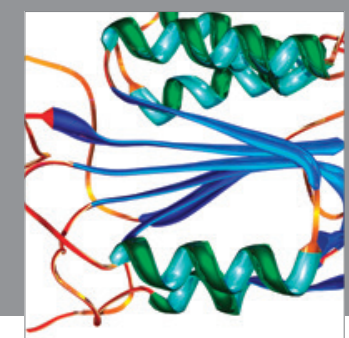

Disease Markers
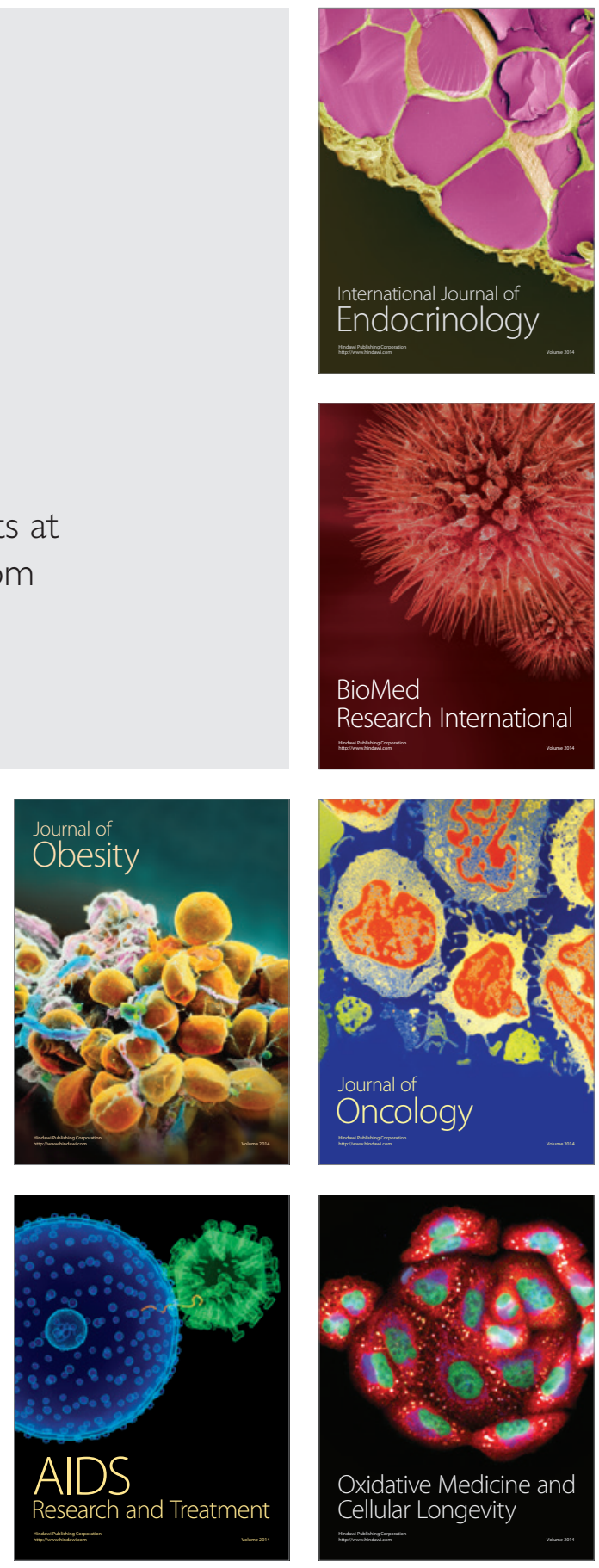